EPJ Web of Conferences 28, 01001 (2012)

DOI: $10.1051 /$ epjconf/20122801001

(C) Owned by the authors, published by EDP Sciences, 2012

\title{
Particle Physics in a Season of Change
}

\author{
Chris Quigga \\ Fermi National Accelerator Laboratory, P.O. Box 500, Batavia, Illinois 60510 USA
}

FERMILAB-CONF-12-035-T

Abstract. A digest of my opening remarks at the 2011 Hadron Collider Physics Symposium.

\section{Introduction}

I have chosen my title to reflect the transitions we are living through, in particle physics overall and in hadron collider physics in particular. Data-taking has ended at the Tevatron, with $\sim 12 \mathrm{fb}^{-1}$ of $\bar{p} p$ interactions delivered to CDF and $\mathrm{D} \emptyset$ at $\sqrt{s}=1.96 \mathrm{TeV}$. The Large Hadron Collider has registered a spectacular first full-year run, with ATLAS and CMS seeing $>5 \mathrm{fb}^{-1}$, LHC $b$ recording $\sim 1 \mathrm{fb}^{-1}$, and ALICE logging nearly $5 \mathrm{pb}^{-1}$ of $p p$ data at $\sqrt{s}=7 \mathrm{TeV}$, plus a healthy dose of $\mathrm{Pb}-\mathrm{Pb}$ collisions. The transition to a new energy regime and new realms of instantaneous luminosity exceeding $3.5 \times 10^{33} \mathrm{~cm}^{-2} \mathrm{~s}^{-1}$ has brought the advantage of enhanced physics reach and the challenge of pile-up reaching $\sim 15$ interactions per beam crossing.

I am happy to record that what the experiments have (not) found so far has roused some of my theoretical colleagues from years of complacency and stimulated them to think anew about what the TeV scale might hold. We theorists have had plenty of time to explore many proposals for electroweak symmetry breaking and for new physics that might lie beyond established knowledge. With so many different theoretical inventions in circulation, it is in the nature of things that most will be wrong. Keep in mind that we learn from what experiment tells us is not there, even if it is uncommon to throw a party for ruling something out. Some non-observations may be especially telling: the persistent absence of flavor-changing neutral currents, for example, seems to me more and more an important clue that we have not yet deciphered.

It is natural that the search for the avatar of electroweak symmetry breaking preoccupies participants and spectators alike. But it is essential to conceive the physics opportunities before us in their full richness. I would advocate a three-fold approach: Explore, Search, Measure! The first

\footnotetext{
a e-mail: quigg@fnal.gov
}

phase of running at the LHC has brought us to two new lands - in proton-proton and lead-lead collisions-and we may well enter other new lands with each change of energy or increase of sensitivity. I believe that it will prove very rewarding to spend some time simply exploring each new landscape, without strong preconceptions, to learn what is there and, perhaps, to encounter interesting surprises. Directed searches, for which we have made extensive preparations, are of self-evident interest. Here the challenge will be to broaden the searches over time, so the searches are not too narrowly directed. Our very successful conception of particles and forces is highly idealized. We have a great opportunity to learn just how comprehensive is our network of understanding by making precise measurements and probing for weak spots, or finding more sweeping accord between theory and experiment.

Indeed, one of the strengths of our position coming into the new era is that over the past few decades, we have conceived, elaborated, and validated two new laws of nature, quantum chromodynamics and the electroweak theory. These gauge theories derive interactions among pointlike $\left(r \lesssim 10^{-18} \mathrm{~m}\right)$ quarks and leptons from the $\mathrm{SU}(3)_{\mathrm{c}} \otimes$ $\mathrm{SU}(2)_{\mathrm{L}} \otimes \mathrm{U}(1)_{Y}$ symmetry inferred from experimental observations. How the $\mathrm{SU}(2)_{\mathrm{L}} \otimes \mathrm{U}(1)_{Y}$ symmetry is hidden is the most urgent question we face. Let us briefly consider the two components of the standard-model interactions.

\section{Quantum Chromodynamics}

A defining feature of QCD is that it is an asymptotically free theory in which the running coupling decreases with increasing scale, or decreasing distance [1]. Indeed, experiments have established that $\alpha_{\mathrm{s}}(Q)$ decreases from a bit less than $1 / 3$ in the neighborhood of $Q=2 \mathrm{GeV}$ to less than $1 / 9$ near $Q=200 \mathrm{GeV}$. It is this property that 
reconciles the success of the parton model with the nonobservation of free quarks, and enables the many successes of perturbative calculations, now reaching to $Q \approx 1 \mathrm{TeV}$. Notable examples are the agreement between theory and experiment for $p^{ \pm} p \rightarrow$ dijets $+X$ over roughly nine orders of magnitude in cross sections and the resemblance to Rutherford scattering that tests both QCD dynamics and the elementary character of quarks. Thanks to progress in lattice gauge theory, we have also achieved a growing understanding of the nonperturbative regime. Calculations of the light-hadron masses that incorporate the influence of quark-antiquark pairs reproduce the spectrum within a few percent and establish that color confinement-i.e., QCDexplains nearly all of the nucleon mass.

From the point of view of mathematical self-consistency, QCD could be complete up to the Planck scale, but that doesn't prove that it is. While the theory exhibits no structural defects, we still do not have an established solution to the strong CP problem. Even as we regard QCD as a solid basis for calculating backgrounds and signals, we need to prepare for surprises. If we ask how QCD might crack, we might imagine breakdowns of factorization (which would compromise our ability to make reliable perturbative calculations), the observation of free quarks or unconfined color, novel kinds of colored matter, quark compositeness, or a larger symmetry containing QCD.

It is arguably more likely that we will encounter new phenomena within QCD. Examination of ("soft") multiparticle production might reveal additional components beyond the established diffraction plus short-range-order. The expected high density of few-GeV partons may have several novel consequences, including thermalization (perhaps revealed in high-multiplicity events) and events containing many minijets. Long-range correlations may emerge. I suspect that a few percent of "minimum-bias" events may exhibit unusual event structures, with the "few percent" increasing with $\sqrt{s}$ and charged-particle multiplicity. Bjorken has suggested that we might be able to recognize collisions involving different configurations of the valence quarks in the proton. To cite a single example, a quark-diquark body plan for the proton might imply diquark-diquark collisions with characteristics different from the familiar quark-quark collisions. Scanning event displays tailored to the anticipated dynamics of multiple production may be an effective way to explore for hints of new phenomena [2].

ALICE, ATLAS, and CMS have opened a new chapter of heavy-ion physics at the LHC. The clear signs of jet quenching [3] and indications of quarkonium $(\Upsilon)$ melting [4] are only the first indications of a rich field of study to come. My formula, Explore, Search, Measure! applies to heavy-ion collisions as well.

\section{The Electroweak Theory}

To good approximation, the electroweak theory is specified by a three-generation V - A structure for the chargedcurrent weak interactions. Flavor-changing neutral currents are suppressed by the Glashow-Iliopoulos-Maiani mechanism, and the (Cabibbo-Kobayashi-Maskawa) quark-mixing matrix describes $\mathrm{CP}$ violation [5].

Although the underlying $\mathrm{SU}(2)_{\mathrm{L}} \otimes \mathrm{U}(1)_{Y}$ gauge symmetry is broken to $\mathrm{U}(1)_{\mathrm{EM}}$, LEP experiments have tested the full symmetry by measuring the cross section for the reaction $e^{+} e^{-} \rightarrow W^{+} W^{-}$and confirming the intricate gaugesymmetry cancellation among the $v_{e^{-}}, \gamma$-, and $Z^{0}$-exchange diagrams [6]. Assiduous study of quantum corrections to many observables led to the inference that the top-quark mass must lie in the interval $150 \mathrm{GeV} \lesssim m_{t} \lesssim 200 \mathrm{GeV}$.

Overall, the accord between the electroweak theory and observations is highly impressive, but perhaps the agreement is not perfect [7]. Among persistent tensions in $B$ sector, it is worth noting the divergence among inclusive, exclusive, and annihilation determinations of the quarkmixing matrix element $\left|V_{u b}\right|$. An amusing suggestion is that the three different values could be brought into agreement by allowing for a small right-handed $u \leftrightarrow b$ interaction [8].

An unknown agent provocateur hides the electroweak symmetry. One of our prime goals is to identify that agent. The possibilities include $\square$ a force of a new character, based on interactions of an elementary scalar (the Higgs boson of the standard model); $\square$ a new gauge force, perhaps acting on undiscovered constituents; $\square$ a residual force that emerges from strong dynamics among electroweak gauge bosons; $\square$ an echo of extra spacetime dimensions.

The electroweak theory does not predict the mass of the Higgs boson, but a simple thought experiment identifies a tipping point, or conditional upper bound on $M_{H}$. The lowest-order diagrams for scattering of $W^{+} W^{-}, Z^{0} Z^{0}, H H$, and $H Z^{0}$ satisfy $s$-wave unitarity, provided that

$$
M_{H}<\left(\frac{8 \pi \sqrt{2}}{3 G_{\mathrm{F}}}\right)^{1 / 2} \approx 1 \mathrm{TeV}
$$

If this bound is respected, perturbation theory is reliable (except near particle poles) and the Higgs boson can be observed on the $1-\mathrm{TeV}$ scale. If not, the weak interactions among $W^{ \pm}$and $Z^{0}$ become strong on $1-\mathrm{TeV}$ scale. Either way (and this important conclusion holds beyond the standard electroweak theory), new physics is to be found on the $1-\mathrm{TeV}$ scale. Within the standard model, analyses of quantum corrections favor $114 \mathrm{GeV} \lesssim M_{H} \lesssim 143 \mathrm{GeV}$ at 95\% confidence level [9]. These studies confirm that something like the Higgs boson couples to $W^{+} W^{-}$and $Z^{0} Z^{0}$ as prescribed by the electroweak theory, but they are insensitive to Higgs-boson couplings to fermions. At this moment, we do not know that the agent of electroweak sym- 
metry breaking gives mass to fermions. Even if the standard electroweak theory should turn out be correct in every particular, we do not know what determines fermion masses and mixings. No calculation within the electroweak theory yields the mass of the electron, or of the top quark, or any relations among quark and lepton masses. I regard all the quark and lepton masses as evidence for physics beyond the standard model.

Within the next year, our experiments may gain the sensitivity needed to pronounce on the existence or nonexistence of the standard-model Higgs boson. That will be a monumental moment in the development of our science, and we must be prepared to explain the motivation and consequences as accurately, completely, and engagingly as we can. That means, first, that you are personally responsible for getting the science right. Some of our colleagues (and too many science writers) persist in repeating the manifestly false statement that the Higgs boson is responsible for all mass, when it is QCD that explains most of the visible mass of the universe in the form of nucleon masses. You are also personally responsible for not propagating made-up history. The people [10] to whom we owe the marvelous insight that spontaneous breaking of a gauge symmetry leads to massive gauge bosons did not set out to understand why matter (in the form of elementary fermions) has mass, and they had nothing to say about the weak interactions. Both the spontaneously broken $\mathrm{SU}(2)_{\mathrm{L}} \otimes \mathrm{U}(1)_{Y}$ theory and the notion that fermion mass could arise from Yukawa couplings of the Higgs scalar to the fermions appear for the first time in Weinberg's 1967 paper [11]. If you owe your acquaintance with the history of the electroweak theory to hearsay or to la presse people, you can find a reliable narrative in Ref. [12]. The real stories are more powerful than fictional cartoons.

We also have an obligation to explain why the search for the agent of electroweak symmetry breaking justifies the resources invested in the LHC and the experiments, not to mention your own time and energy. One approach is to ask how different the world would have been, without a Higgs mechanism or a substitute on the real-world electroweak scale [13]. Think, for simplicity, of one generation of fermions. Without a Higgs vacuum expectation value, the electron and quarks would have no mass. Eliminating the Higgs mechanism does not alter the strong interaction, so QCD would still confine colored objects into hadrons. The gross features of nucleons derived from QCD—such as nucleon masses-would be little changed if the up and down quark masses vanished. If the quarks are massless, the QCD Lagrangian displays an $\mathrm{SU}(2)_{\mathrm{L}} \otimes \mathrm{SU}(2)_{\mathrm{R}}$ chiral symmetry that is spontaneously broken near the confinement scale to isospin symmetry by the formation of $\langle\bar{q} q\rangle=$ $\left\langle\bar{q}_{\mathrm{L}} q_{\mathrm{R}}\right\rangle+\left\langle\bar{q}_{\mathrm{R}} q_{\mathrm{L}}\right\rangle$ condensates. These condensates couple left-handed and right-handed quarks, giving rise to the effective "constituent-quark" masses and breaking the elec- troweak symmetry because left-handed and right-handed quarks transform differently under $\mathrm{SU}(2)_{\mathrm{L}} \otimes \mathrm{U}(1)_{Y}$. The weak bosons $W$ an $Z$ acquire masses, but they are 2500 times smaller than in the real world: the analogue of the Fermi constant, $G_{\mathrm{F}}$, is enhanced by nearly seven orders of magnitude. Should the proton be stable, or compound nuclei be produced and survive to late times in this alternate universe, the infinitesimal electron mass would compromise the integrity of matter. The Bohr radius of a would-be atom would be macroscopic (if not infinite), so an electron could not be associated with a specific nucleus and valence bonding would have no meaning. Seeking the agent of electroweak symmetry breaking, we hope to learn why the everyday world is as we find it: why atoms and chemistry and stable structures such as liquids and solids can exist.

Returning to this world, let us suppose that the agent of electroweak symmetry breaking is indeed a light, elementary scalar. Then we will be forced to ask whether $M_{H}<1 \mathrm{TeV}$ makes sense in the framework of quantum field theory. It seems inevitable that if the ultimate theory contains meaningful distant scales - a unification scale or the Planck scale, for example - quantum corrections would tend to pull the Higgs-boson mass up to far higher scales than $1 \mathrm{TeV}$, unless it is stabilized by a symmetry or a dynamical principle. This is the essence of the hierarchy problem. Supersymmetry could control the quantum corrections by balancing bosonic and fermionic loop contributions. If dynamical symmetry breaking should yield a light Higgs Doppelgänger (with or without fermion couplings), the composite nature of that stand-in would also damp quantum shifts and bring them under control.

Despite the hints we have for a light "Higgs boson," we have not seen evidence for the new symmetry or dynamics that might serve to make a low mass natural. We can state the mystery in the form of two puzzles:

II Puzzle \#1: We expect "New Physics" on the 1-TeV scale to stabilize Higgs mass and solve the hierarchy problem, but there is no sign of the flavor-changing neutral currents that occur generically in extensions to the standard model. The notion of minimal flavor violation, that the structure of the quark-mixing matrix controls all flavor phenomena, is a name, but not yet an answer. Accordingly, there is great interest in searches for forbidden or suppressed processes that might reveal something about flavor-changing neutral currents.

II Puzzle \#2: We expect "New Physics" on the 1-TeV scale to stabilize Higgs mass and solve the hierarchy problem, but experiment has not established a pattern of serious quantitative failures of electroweak theory.

To these two puzzles, which have been growing in significance since the LEP era, we may add the observation that no departures from established physics have turned up in early running of the LHC. Supersymmetry, in particular, 


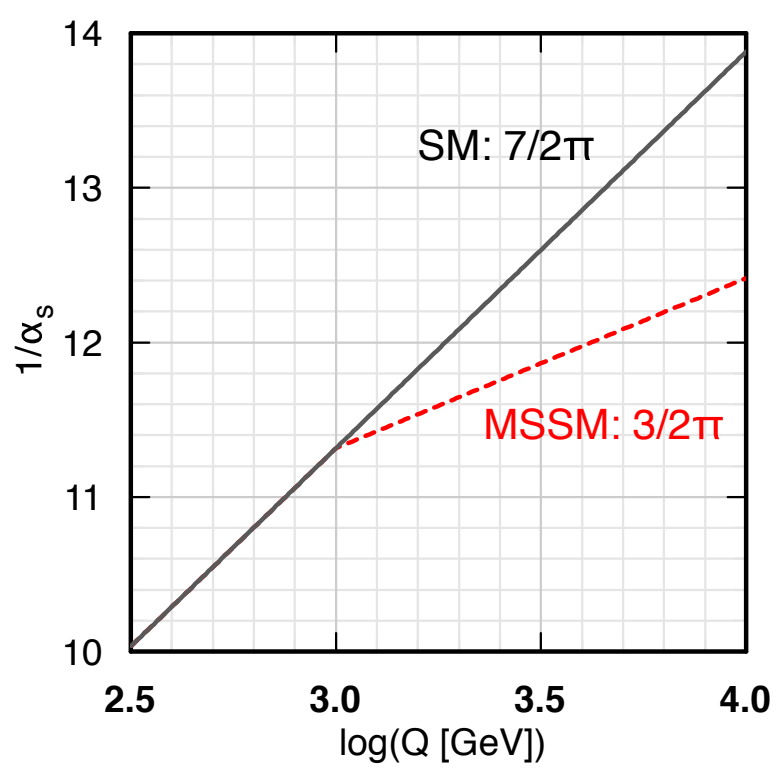

Fig. 1. Evolution of the strong coupling constant (displayed as $1 / \alpha_{\mathrm{s}}$ ) in the standard and supersymmetric versions of the $\mathrm{SU}(5)$ unified theory, for superpartners that become active at $Q=1 \mathrm{TeV}$.

is hiding very effectively. Perhaps it is time to ask whether the unreasonable effectiveness of the standard model (to borrow a turn of phrase from Wigner [14]) is itself a deep clue to what might lie beyond the standard model.

One of the ambitious hopes for a more comprehensive takes the form of a unified theory that encompasses the $\mathrm{SU}(3)_{\mathrm{c}} \otimes \mathrm{SU}(2)_{\mathrm{L}} \otimes \mathrm{U}(1)_{Y}$ standard model. Simple examples of unified theories foresee a unification of forces in which the (suitably normalized) $\mathrm{SU}(3)_{\mathrm{c}}, \mathrm{SU}(2)_{\mathrm{L}}$, and $\mathrm{U}(1)_{Y}$ coupling constants evolve toward a common value at some high unification scale. Calculations in perturbation theory, applied to the measured low-energy values of the couplings, show that coupling-constant unification is more promising in supersymmetric SU(5) than in the original SU(5) theory, provided that the change in evolution due to a full spectrum of superpartners occurs near $1 \mathrm{TeV}$.

It is interesting to ask whether LHC experiments could test this hypothesis by measuring the strong coupling constant $\alpha_{\mathrm{s}}$ or the weak mixing parameter $\sin ^{2} \theta_{\mathrm{W}}$ as functions of scale. I sketch in Figure 1 the evolution of $1 / \alpha_{\mathrm{s}}$, in leading logarithmic approximation, with and without a superpartner threshold at $Q=1 \mathrm{TeV}$. The slope changes significantly, from $7 / 2 \pi$ to $3 / 2 \pi$, at what I have taken here as a sharp threshold. Seeing, or not seeing, such a change would be powerful evidence for or against the existence of a new set of colored particles that would complement ongoing searches for specific new-particle signatures.

Considerable work will be required to determine promising classes of measurements. I suspect that the study of

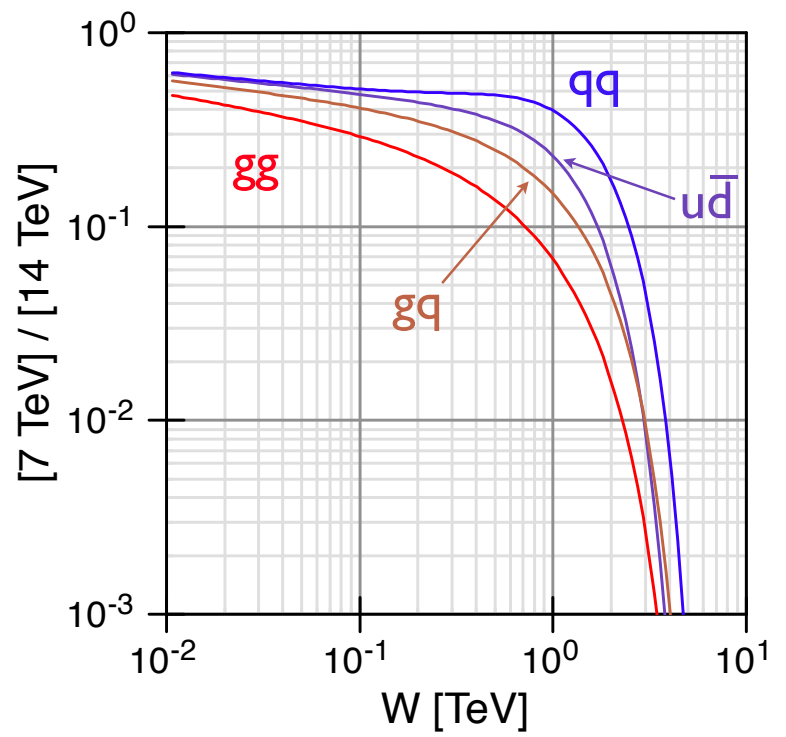

Fig. 2. Ratios of parton luminosities in $p p$ collisions at $\sqrt{s}=$ $7 \mathrm{TeV}$ and $14 \mathrm{TeV}$. At $W=1 \mathrm{TeV}$, the curves from top to bottom refer to $q q, u \bar{d}, g q$, and $g g$, where $q$ is a light ( $u$ or $d$ ) quark. The CTEQ6L1 parton distributions are used. (From Ref. [15].)

$Z^{0}+$ jets will be fruitful. A continuing dialogue between theory and experiment will be needed to isolate $\alpha_{\mathrm{s}}(Q)$ measured at a high scale.

\section{4 À suivre...}

The last events have been recorded at the Tevatron collider, but the interpretation of data, now enriched by conversation with LHC experiments, continues. I look forward to a rich year of final results from $\mathrm{CDF}$ and $\mathrm{D} \varnothing$, ranging from searches for the standard-model Higgs boson to legacy measurements of the $W$-boson mass. The LHC experiments have made wonderful beginnings on the three fronts-Explore, Search, Measure! - but we have miles to go. The impressive luminosities delivered to ALICE, ATLAS, CMS, and LHC $b$ prefigure the much larger data samples to come, and the LHC is operating at only half its design energy. I show in Figure 2 the ratios of selected parton luminosities at $\sqrt{s}=7 \mathrm{TeV}$ to those at $\sqrt{s}=14 \mathrm{TeV}$, as a function of the parton-parton subenergy $W$. Even in the $100-\mathrm{GeV}$ range, the advantage of $14 \mathrm{TeV}$ over $7 \mathrm{TeV}$ is noticeable. For $W \gtrsim 1 \mathrm{TeV}$, the advantage of higher-energy running becomes decisive.

Here are some of the questions on my mind:

1. What is the agent of electroweak symmetry breaking? Is there a Higgs boson? Might there be several?

2. Is the Higgs boson elementary or composite? How does it interact with itself? What shapes the Higgs potential- 
or, more generally, triggers electroweak symmetry breaking?

3. Does the Higgs boson give mass to fermions, or only to the weak bosons? What sets the masses and mixings of the quarks and leptons? (How) is fermion mass related to the electroweak scale?

4. Will new flavor symmetries give insights into fermion masses and mixings?

5. What stabilizes the Higgs-boson mass below $1 \mathrm{TeV}$ ?

6. Do the different charged-current behaviors of left-handed and right-handed fermions reflect a fundamental asymmetry in nature's laws?

7. What will be the next symmetry we recognize? Are there additional heavy gauge bosons? Is nature supersymmetric? Is the electroweak theory contained in a grander unified theory?

8. Are all flavor-changing interactions governed by the standard-model Yukawa couplings? Does "minimal flavor violation" hold? If so, why? At what scale?

9. Are there additional sequential quark and lepton generations? Or new exotic (vector-like) fermions?

10. What resolves the strong CP problem?

11. What are the dark matters? Might dark matter have a flavor structure?

12. Is electroweak symmetry breaking an emergent phenomenon connected with strong dynamics? How would that alter our conception of unified theories of the strong, weak, and electromagnetic interactions?

13. Is electroweak symmetry breaking related to gravity through extra spacetime dimensions?

14. What resolves the vacuum energy problem?

15. (When we understand the origin of electroweak symmetry breaking,) what lessons will electroweak symmetry breaking hold for unified theories? ... for inflation? ... for dark energy?

16. Will experiments reveal unexpected phenomena in strong interactions?

17. What explains the baryon asymmetry of the universe? Are there new CP-violating phases in charged-current interactions?

18. Are there new flavor-preserving phases? What would observation, or more stringent limits, on electric-dipole moments imply for theories beyond the standard model?

19. (How) are quark-flavor dynamics and lepton-flavor dynamics related (beyond the gauge interactions)?

20. At what scale are the neutrino masses set? Is the neutrino its own antiparticle?

$$
\ldots \text { and finally ... }
$$

21. How are we prisoners of conventional thinking?

We should have much to digest — and to celebrate-at HCP 2012 in Kyoto. In anticipation of another intense year, I leave you with the words of Stewart Brand [16]:

Stay hungry. Stay foolish.

\section{Acknowledgements}

Fermilab is operated by Fermi Research Alliance, LLC under Contract No. DE-AC02-07CH11359 with the United States Department of Energy. I thank Gregorio Bernardi and the Organizing Committee for the invitation to speak, and thank all participants for a lively and stimulating symposium.

\section{References}

1. For an extensive QCD bibliography with illustrations of the principal consequences and tests, see A. S. Kronfeld and C. Quigg, Am. J. Phys. 78 (2010)10811116.

2. C. Quigg, "Looking into Particle Production at the Large Hadron Collider," Nuovo Cim. 33C (2011) 327333 [arXiv:1004.0975].

3. G. Aad, et al. [Atlas Collaboration], Phys. Rev. Lett. 105 (2010) 252303; S. Chatrchyan. et al. [CMS Collaboration], Phys. Rev. C84 (2011) 024906.

4. S. Chatrchyan, et al. [CMS Collaboration], Phys. Rev. Lett. 107 (2011) 052302.

5. For a digest of the electroweak theory, extensive citations to the experimental tests, and unanswered questions, see C. Quigg, Annu. Rev. Nucl. Part. Sci. 59 (2009) 505-555.

6. A summary of the evidence can be found at the LEP Electroweak Working Group web site, http://lepewwg.web.cern.ch/LEPEWWG/.

7. E. Lunghi and A. Soni, "Demise of CKM and its aftermath," arXiv:1104.2117.

8. For one example, see A. J. Buras, K. Gemmler and G. Isidori, Nucl. Phys. B 843 (2011) 107.

9. For an example of constraints on the mass of the standard-model Higgs boson and other parameters, see H. Flächer, et al. [Gfitter Collaboration], Eur. Phys. J. C 60, 543 (2009) [Erratum-ibid. C 71, 1718 (2011)]; M. Baak, et al., "Updated Status of the Global Electroweak Fit and Constraints on New Physics," arXiv:1107.0975; http://gfitter.desy.de.

10. F. Englert and R. Brout, Phys. Rev. Lett. 13 (1964) 321; P. W. Higgs, Phys. Rev. Lett. 13 (1964) 508, Phys. Lett. 12 (1964) 132; G. S. Guralnik, C. R. Hagen, and T. W. B. Kibble, Phys. Rev. Lett. 13 (1964) 585. The link between gauge invariance and the Meissner effect is made in P. W. Anderson, Phys. Rev. 110 (1958) 827.

11. S. Weinberg, Phys. Rev. Lett. 19 (1967) 1264. For a related development, see A. Salam, in Elementary Particle Theory: Relativistic Groups and Analyticity (Nobel Symposium No. 8), edited by N. Svartholm, Almqvist and Wiksell, Stockholm, 1968, p. 367 [http://j.mp/r9dJOo]. The theory is built on 
the $\mathrm{SU}(2)_{\mathrm{L}} \otimes \mathrm{U}(1)_{Y}$ gauge symmetry investigated by S. L. Glashow, Nucl. Phys. 22 (1961) 579.

12. F. Close, The Infinity Puzzle (Oxford University Press, Oxford, 2011).

13. C. Quigg and R. Shrock, Phys. Rev. D79 (2009) 096002.

14. Compare E. P. Wigner, "The Unreasonable Effectiveness of Mathematics in the Natural Sciences." Commun. Pure Appl. Math. 13 (1960) 1-14.

15. C. Quigg, "LHC Physics Potential vs. Energy," arXiv:0908.3660; "LHC Physics Potential vs. Energy: Considerations for the 2011 Run," arXiv:1101.3201. See also the archives of figures at http://lutece.fnal.gov/PartonLum/ (various LHC energies compared with $2-\mathrm{TeV} \bar{p} p$ and $14-\mathrm{TeV} p p$ ) and http://lutece.fnal.gov/PartonLum11/ (emphasizing the comparison of $p p$ at $\sqrt{s}=8 \mathrm{TeV}$ and $7 \mathrm{TeV}$ ).

16. S. Brand, The Whole Earth Epilog (Point Foundation, San Francisco, 1974). 